\title{
Influência da Ordem dos Exercícios Sobre o Número de Repetições e Percepção Subjetiva do Esforço em Mulheres Jovens e Idosas
}

\author{
Influence of Exercise Order on the Number of Repetitions and \\ Perceived Exertion in Young and Older Women
}

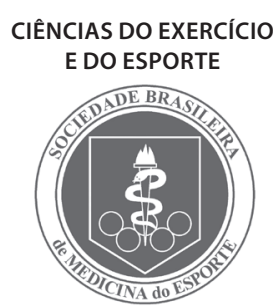

Artigo Original
Nádia Souza Lima da Silva $a^{1,2}$

Walace David Monteiro 2,3

Paulo de Tarso Veras Farinatti2,3

1. Programa de Pós-Graduação em Educação Física - Universidade Gama Filho (UGF).

2. Laboratório de Atividade Física e Promoção da Saúde - Universidade do Estado do Rio de Janeiro (UERJ). 3. Programa de Pós-Graduação em Ciências da Atividade Física - Universidade Salgado de Oliveira (Universo).

Endereço para correspondência: Nadia Souza Lima da Silva ou Paulo Farinatti

Universidade do Estado do Rio de Janeiro (LABSAU-UERJ).

Rua São Francisco Xavier 524, 8oㅡ and., sala 8.133, Bloco F.

20599-900 - Rio de Janeiro, RJ.

E-mail: nadialima@globo.com ou farinatt@uerj.br

Submetido em 26/03/2008 Versão final recebida em 16/09/2008 Aceito em 28/11/2008

\begin{abstract}
RESUMO
Estudos sobre a influência da ordem de execução dos exercícios no desempenho da força em idosos são escassos. O objetivo do presente estudo foi comparar a influência da ordem de execução dos exercícios sobre o número de repetições e percepção de esforço de oito idosas (69 \pm 7 anos) e 12 jovens (22 \pm 2 anos) praticantes de treinamento resistido. As voluntárias executaram sessões com três séries até a exaustão com cargas estabelecidas em 10 repetições máximas (RM), em sequências invertidas de exercícios: sequência $A$ - supino horizontal (SH), desenvolvimento em pé (DP) e rosca tríceps no pulley baixo (TP); sequência B - TP, DP e SH. A ANOVA de duas entradas com medidas repetidas revelou que o número de repetições no grupo de jovens foi sempre menor no último exercício, independentemente da sequência ou grupamento muscular envolvido $(p<0,05)$. O número de repetições permaneceu estável na sequência A para grupo de idosas, mas declinou significativamente na sequência $B(p<0,05)$. Não houve diferenças para a percepção de esforço ao final das sequências no grupo de jovens, mas no grupo de idosas a PSE foi significativamente maior em na sequência $B(p<0,05)$. Conclui-se que a ordem dos exercícios foi indiferente para o desempenho do grupo jovem, mas influenciou o número máximo de repetições em cada exercício e a percepção de esforço ao final das sequências no grupo de idosas.
\end{abstract}

Palavras-chave: envelhecimento, treinamento, força muscular, exercício, saúde, aptidão física.

\begin{abstract}
There are few studies about the effects of exercise order on strength training performance in elderly subjects. This study compared the influence of exercise order in resistive training on the number of repetitions and perceived exertion of 8 older ( $69 \pm 7$ years) and 12 young women (22 \pm 2 years). The subjects performed three sets until exhaustion with loads established at 10 maximum repetitions (10 RM) with inverted sequences of the following exercises: Sequence $A$ - bench press (BP), military press (MP), pulley triceps extension (TP); Sequence B - TP, MP, and BP. The two-way ANOVA for repeated measurements showed that the number of repetitions in the young group was always smaller for the last exercise, regardless of the sequence or muscle group involved $(p<0.05)$. The number of repetitions was not different in Sequence A for the older group, but declined significantly in Sequence B $(p<0.05)$. Perceived exertion was similar in both sequences for the young group, but in the older group it was significantly higher in Sequence $B(p<0.05)$. In conclusion, exercise order was indifferent for the young group performance, but influenced in the maximum number of repetitions in each exercise and perceived exertion at the end of the sequences in the older group.
\end{abstract}

Keywords: aging, training, strength, exercise, health, physical fitness.

\section{INTRODUÇÃO}

O treinamento contrarresistência (TCR) induz aprimoramentos nas funções e estruturas muscular, articular e óssea, aumentando a aceitação de que esses tipos de programas são importantes para a conservação da capacidade de trabalho e qualidade de vida do idoso ${ }^{(1-5)}$. Sabe-se, porém, que os efeitos promovidos pelo TCR dependem da manipulação de diversas variáveis ${ }^{(6,7)}$. Nesse sentido, é a combinação do número de repetições, séries, sobrecarga, intervalos, sequência dos exercícios e velocidade de execução dos movimentos que determina os ganhos de força ${ }^{(8,9)}$.

Silva e Farinatti(10), após revisão sistemática sobre os efeitos das variáveis de prescrição nas respostas ao TCR, identificaram lacunas importantes em relação à manipulação das variáveis de prescrição no que toca à estratégia mais eficiente para programas que visem o ganho de força em idosos. Dentre as lacunas identificadas, menciona-se particu- larmente a ausência de estudos que se preocuparam com a influência da ordenação dos exercícios sobre o desempenho de idosos no TCR.

De forma geral, estudos prévios sugerem que a fadiga parece ser similar em sequências começando por grandes e pequenos grupamentos musculares, manifestando-se sempre mais fortemente nos exercícios realizados ao final de uma sequência(11-13). Logo, a ordenação dos exercícios em uma sessão deveria levar em conta o objetivo central do programa e o estado de treinamento do praticante. No entanto, esses estudos foram desenvolvidos com adultos jovens. Não se sabe até que ponto seus resultados poderiam ser transferidos para indivíduos idosos, uma vez que a idade se associa a um declínio gradativo das capacidades motoras, em especial da força muscular ${ }^{(14,15)}$. As respostas de fadiga observadas em pessoas idosas, portanto, não necessariamente serão similares àquelas exibidas por indivíduos jovens. 
Entre as formas de combinar a execução dos exercícios em uma sessão de treinamento, diversas opções de ordenação podem ser aplicadas. Em recente investigação sobre o assunto, Gentil et al.(16) sugeriram que as combinações de exercícios mais populares consistem nas técnicas de pré-exaustão e prioritária. Para Fleck e Kraemer ${ }^{(7)}$, a técnica de pré-exaustão caracteriza-se por utilizar um dado grupo muscular, iniciando-se por um exercício monoarticular, passando para um multiarticular. Já a técnica de prioridade consiste em utilizar no início da sessão os exercícios mais relacionados com os objetivos dos praticantes, para que sejam executados com intensidades máximas devido aos menores níveis de fadiga. Nesse caso, não importa o fato de o exercício que inicia a sessão ser mono ou multi-articular.

A técnica que vem recebendo maior atenção na literatura é a de pré-exaustão, talvez devido às maiores possibilidades de induzir fadiga durante a sessão de treinamento ${ }^{(16,17)}$. Nota-se, porém, que os estudos supracitados observaram o efeito da pré-exaustão sobre o desempenho da força com indivíduos jovens. Não existem estudos similares com praticantes idosos, o que se justificaria em virtude das possíveis diferenças de treinabilidade associadas à idade.

Assim, o presente estudo teve por objetivo comparar o número máximo de repetições para uma dada carga e a percepção subjetiva de esforço, em mulheres jovens e idosas, após séries múltiplas de exercícios para membros superiores organizados em ordem inversa. Testou-se a hipótese de que, uma vez mais propensas à fadiga localizada, as mulheres idosas seriam mais sensíveis à manipulação da ordem dos exercícios do que as mais jovens.

\section{MÉTODOS}

\section{Amostra}

A amostra foi composta por oito mulheres idosas ( $69 \pm 7$ anos; $65 \pm$ $8 \mathrm{~kg} ; 154 \pm 6 \mathrm{~cm}$ ) e 12 jovens ( $22 \pm 2$ anos; $64 \pm 11 \mathrm{~kg} ; 166 \pm 7 \mathrm{~cm} ; 22 \pm$ $2 \%$ de gordura), com experiência respectiva de pelo menos três e seis meses na realização de TCR. Como critério de exclusão foi considerado o diagnóstico de problemas osteomioarticulares, cardiovasculares, metabólicos ou cognitivos que pudessem impedir a realização dos exercícios propostos. Antes da coleta de dados todas responderam ao questionário PAR-Q(18) e assinaram termo de consentimento pós-informado, conforme resolução 196/96 do Conselho Nacional de Saúde. O estudo foi aprovado por Comitê de Ética institucional.

\section{Coleta dos dados}

A escolha dos exercícios deve ser justificada. É importante mencionar que, na maior parte dos casos, as sessões são compostas por exercícios para membros inferiores e superiores. Contudo, esse não é sempre o caso, especialmente quando se está lidando com programas de alta intensidade para sujeitos treinados. No que toca à ordem dos exercícios, considera-se que os efeitos da fadiga acumulada no desempenho ocorram mais provavelmente para uma expressiva quantidade de séries com elevado volume (alta intensidade e muitas repetições) e exercícios envolvendo grupamentos musculares primários e secundários relacionados ${ }^{(16)}$. Outro exemplo pode ser encontrado em Miranda et al. ${ }^{(17)}$ os quais, recentemente, investigaram os efeitos de diferentes intervalos de recuperação na fadiga acumulada em protocolo com séries múltiplas apenas para membros superiores. Farinatti et al. ${ }^{(19)}$, por sua vez, observaram a influência da ordem dos exercícios em protocolo idêntico ao aqui proposto sobre o consumo de oxigênio durante os exercícios, intervalos de recuperação e após a sessão (EPOC), a fim de testar a fadiga acumulada e o gasto calórico total associado a suas sequências.

Apenas para ilustrar, se na vida real a ordem dos exercícios dificilmente influenciaria o desempenho de uma rosca bíceps após um leg-press, este poderia ser um aspecto importante a levar em conta se um supino se segue a ou é precedido de uma rosca tríceps. Enfim, é difícil delinear um programa de três séries com 10RM envolvendo exercícios para o corpo inteiro. Esse tipo de programa usualmente é feito com cargas e repetições submáximas. No presente estudo, nota-se que as voluntárias executaram nove séries de exercícios, cuja ação muscular era de certa forma relacionada, isso até a exaustão volitiva máxima, o que provavelmente permitiu a instalação de fadiga acumulada considerável.

\section{Teste de 10 repetições máximas (10RM)}

Todas as voluntárias estavam habituadas a realizar os exercícios nos equipamentos utilizados. Isso, associado às explicações sobre as condutas nos testes, ajudou a oferecer maior segurança, sobretudo para as idosas realizarem os testes. Visando reduzir a margem de erro nos testes de 10RM, foram adotados os procedimentos descritos a seguir: a) instruções padronizadas foram fornecidas antes do teste; b) os sujeitos foram instruídos sobre a técnica de execução do exercício, durante as diferentes etapas de sua realização; c) o avaliador estava atento quanto à posição adotada pelo sujeito no momento da medida, pois pequenas variações no posicionamento das articulações envolvidas no movimento poderiam acionar outros músculos, levando a interpretações errôneas dos escores obtidos; d) foram definidas as seguintes etapas de execução dos exercícios: posição inicial e desenvolvimento, esta última compreendendo as fases concêntrica e excêntrica da contração. Esse procedimento foi adotado para facilitar a contagem dos movimentos realizados de forma correta; e) estímulos verbais foram realizados a fim de manter alto o nível de estimulação durante a execução dos exercícios; f) os pesos utilizados no estudo foram previamente aferidos em balança de precisão; g) os intervalos entre as tentativas em cada exercício durante o teste de 10RM foram fixados entre dois e cinco minutos. Após a primeira tentativa, as avaliadas descansavam até sentirem-se aptas para realizar outra tentativa com nova carga e assim por diante. Como as voluntárias, independentemente da idade, eram treinadas para realizar 10RM em suas sessões habituais de treinamento, as mesmas estavam adaptadas a essa faixa de intervalo de recuperação entre séries. É importante ressaltar que apenas uma idosa utilizou o limite máximo de intervalo para condução dos testes, mesmo assim na última tentativa em um exercício; h) o incremento das cargas nas diferentes tentativas variou entre 1 a $5 \mathrm{~kg}$, tanto para idosas quanto para jovens, devido à familiaridade de ambas com os exercícios e equipamentos usados nos testes. Em alguns casos, utilizaram-se pesos adicionais de $250 \mathrm{~g}$ para melhor discriminar valores intermediários de cargas; i) após obtenção da carga em um determinado exercício, o exercício seguinte era executado após intervalos não inferiores a 10 min ${ }^{(20)}$. A ordem de entrada para a realização dos testes foi contrabalanceada.

\section{Padronização dos exercícios}

Todos os exercícios foram realizados no aglomerado de oito estações da marca Riguetto ${ }^{\circledast}$ (São Paulo, SP), sendo padronizadas duas fases para a sua execução, conforme descrito a seguir.

Supino horizontal (SH) - a) posição inicial do exercício: em decúbito dorsal, com os braços segurando a barra do equipamento, apresentando 90o de flexão entre braço e antebraço, joelhos e quadris semiflexionados, com os pés sobre o apoio do próprio aparelho; b) desenvolvimento do exercício: a partir da posição inicial, realizava-se a extensão completa dos cotovelos e flexão horizontal dos ombros, retornando à posição inicial. Desenvolvimento em pé (DP) - a) posição inicial do exercício: em pé, de frente para o equipamento, joelhos semiflexionados, com os cotovelos flexionados, de modo que a barra do equipamento ficasse situada logo acima da linha dos ombros. b) desenvolvimento do exercício: a partir da posição inicial, realizava-se a extensão completa dos cotovelos com abdução de ombros. Após o término da fase anterior, os cotovelos eram novamente flexionados e os ombros aduzidos, até a retomada da posição inicial. Tríceps no 
pulley (TP) - a) posição inicial do exercício: em pé, de frente para a polia alta do equipamento, pernas paralelas com um pequeno afastamento lateral com os joelhos semiflexionados, quadris na posição anatômica, cotovelos flexionados, com as mãos pronadas segurando a barra e a cabeça posicionada com o plano de Frankfurt; b) desenvolvimento do exercício: a partir de uma flexão aproximada de 300 entre braço e antebraço, realizava-se extensão completa dos cotovelos e ombros aduzidos. Após finalizada essa fase, os cotovelos eram novamente flexionados até a retomada da posição inicial.

Após a obtenção das cargas máximas no teste de 10RM as voluntárias descansavam por 48 horas e, posteriormente, eram reavaliadas para consecução da reprodutibilidade das cargas obtidas para 10RM (teste e reteste). Para evitar interferência nos resultados, as voluntárias foram orientadas a não realizar nenhum tipo de exercício entre as sessões de testes. A reprodutibilidade das cargas para as repetições previstas foi determinada através de duas medidas. Isso foi possível devido ao fato de todas as voluntárias serem treinadas para realizar nos exercícios escolhidos e, mais ainda, nos equipamentos utilizados no estudo. Além disso, o número de repetições máximas avaliadas também era o mesmo do treinamento habitual das voluntárias, o que facilitou a obtenção de medidas estáveis.

\section{Protocolo de aplicação dos exercícios contrarresistência}

Posteriormente à realização do teste de 10RM, os exercícios selecionados foram feitos em duas ordenações: sequência A (SEQA) - SH, DP e TP; sequência B (SEQB) - TP, DP e SH. A inclusão dos indivíduos na realização das sequências foi feita de forma alternada. Antes de iniciar a sequência era realizado um aquecimento específico no primeiro exercício, com 12 repetições a 40\% de 10RM. É importante ressaltar que, ao longo das séries, pediu-se às voluntárias que realizassem o máximo número de repetições com a carga associada às 10 RM, permitindo-se que houvesse declínio em virtude de falha concêntrica. Esse foi o indicador de desempenho e, portanto, a variável que indicava a influência da ordem dos exercícios nas diferentes sequências investigadas. Além disso, a velocidade de execução não foi padronizada, de forma que ambos os grupos pudessem realizar o número previsto de repetições da melhor forma possível.

Dois minutos após o aquecimento, as três séries de cada exercício eram realizadas com cargas de 10RM até a falha concêntrica do movimento. Entre as séries e exercícios foram fixados três minutos de intervalo. Ao final de cada série e exercício era registrado, o número máximo de repetições realizadas e apresentava-se ao sujeito a escala de Borg CR-10 para indicação da percepção subjetiva de esforço. De acordo com as instruções veiculadas por Borg ${ }^{(21)}$, as voluntárias foram instruídas a enfatizar a percepção localizada do esforço.

\section{Tratamento dos resultados}

A reprodutibilidade das cargas para 10RM foi determinada pelo coeficiente de correlação intraclasse (ICC). A distribuição normal dos dados foi verificada por meio do teste de Shapiro-Wilk. Devido à assimetria e diferença do padrão de distribuição normal, os dados para a percepção subjetiva do esforço foram transformados por meio do cálculo do logaritmo natural de seus valores absolutos para os respectivos ajustes das curvas. Para verificar as diferenças no número de repetições em cada exercício foi utilizada uma ANOVA de duas entradas com medidas repetidas, seguida de verificação post-hoc de Fisher, quando necessário. Os pressupostos de homogeneidade de variâncias e heterocedasticidade foram testados automaticamente antes do cálculo da ANOVA, pelo próprio software estatístico. Para comparação da percepção subjetiva de esforço nas sequências aplicou-se o teste $t$ de Student para amostras pareadas. Em todos os casos, adotou-se nível de significância de $p<0,05$. Os cálculos foram efetuados com auxílio do programa Statistica 6.0 (Statsoft ${ }^{\oplus}$, Tulsa, EUA).

\section{RESULTADOS}

Os valores do ICC para a reprodutibilidade entre as cargas de 10RM revelaram-se satisfatórios nos três exercícios selecionados ${ }^{(22-24)}$. Mulheres idosas: $\mathrm{SH}(\mathrm{ICC}=0,88 ; \mathrm{p}<0,01)$, DP $(\mathrm{ICC}=0,85 ; \mathrm{p}<0,01)$ e TP $(0,91$; $p<0,01)$; mulheres jovens: SH (ICC $=0,91$; $p<0,01)$, DP $(I C C=0,93$; $p<0,01)$, e TP $(I C C=0,94 ; p<0,01)$.

A tabela 1 apresenta os valores encontrados para o número de repetições nas sequências analisadas. Para as mulheres jovens, o número de repetições de SH foi significativamente superior ao de TP em SEQA. O oposto se deu em SEQB. Assim, quando TP foi executado primeiro, mesmo envolvendo o menor grupamento muscular, o número de repetições foi significativamente maior que o observado nos demais exercícios, principalmente em relação ao SH, último da sequência. No grupo de idosas, iniciar a sequência pelo exercício com o maior grupamento muscular pareceu não afetar o desempenho nos demais exercícios (SEQA). Na sequência inversa, porém, o total de repetições em TP e DP revelou-se significativamente maior que o observado em $\mathrm{SH}$.

Tabela 1. Número de repetições totais nas três séries para os exercícios supino horizontal (SH), desenvolvimento em pé (DP) e rosca tríceps (TP) em mulheres idosas e jovens.

\begin{tabular}{|c|c|c|c|}
\hline \multicolumn{4}{|c|}{ Mulheres jovens $(n=12)$} \\
\hline & SH & DS & TP \\
\hline SEQA & $27,8 \pm 3,2^{\mathrm{a}}$ & $27,5 \pm 2,7$ & $23,9 \pm 9,0^{* b}$ \\
\hline SEQB & $20,4 \pm 7,8$ & $28,8 \pm 1,8^{* *}$ & $29,9 \pm 0,4^{* *}$ \\
\hline \multicolumn{4}{|c|}{ Mulheres idosas $(n=8)$} \\
\hline & $\mathrm{SH}$ & DS & TP \\
\hline SEQA & $26,8 \pm 1^{\mathrm{a}}$ & $26,4 \pm 3,5$ & $27,2 \pm 0,9$ \\
\hline SEQB & $21,0 \pm 2,4$ & $27,3 \pm 1,9$ & $27,7 \pm 0,8^{* *}$ \\
\hline
\end{tabular}

A figura 1 apresenta os valores médios para a escala de Borg (CR-10) ao final de cada sequência. Não houve diferenças para o grupo de jovens para a percepção do esforço entre as sequências $(p=0,42)$. Em contrapartida, o grupo idoso teve percepção significativamente inferior àquela das jovens em SEQA $(p=0,03)$, a qual também experimentou elevação significativa em SEQB $(p=0,02)$.

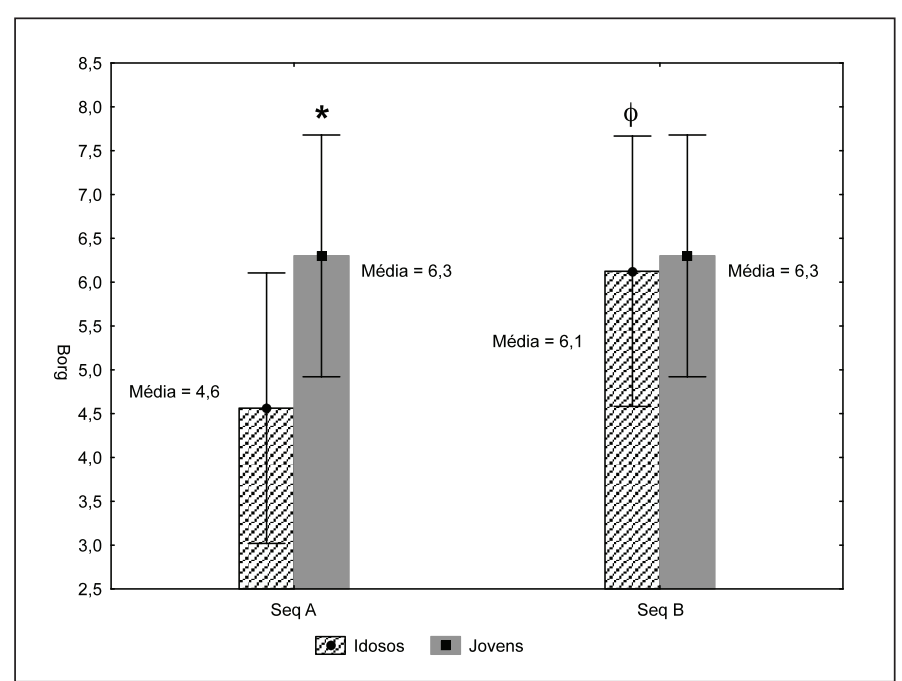

Figura 1. Percepção subjetiva do esforço para jovens e idosas nas sequências de exercícios investigadas quantificada pela escala de Borg CR-10. *diferença significativa entre jovens e idosas na SEQA. $\phi$ Diferença significativa entre SEQA e SEQB para o grupo de idosas $(p<0,05)$. As barras indicam os intervalos de confiança para $95 \%$. 


\section{DISCUSSÃO}

O objetivo do presente estudo foi comparar o número máximo de repetições para uma dada carga e a percepção subjetiva de esforço, em mulheres jovens e idosas, após séries múltiplas de exercícios para membros superiores organizados em ordem inversa. Os resultados indicaram que o número de repetições no grupo de jovens foi sempre menor no último exercício, independentemente da sequência ou grupamento muscular envolvido, enquanto que permaneceu estável na sequência A para o grupo de idosas, declinando significativamente na sequência iniciada pelo menor grupamento muscular (SEQB) $(p<$ 0,05). Não houve diferenças para a percepção de esforço ao final das sequências no grupo de jovens, mas no grupo de idosas tendeu a ser maior para SEQB em comparação com SEQA.

Para analisar os efeitos do esforço associado a uma sessão de TCR, deve-se confiar na intensidade das cargas utilizadas, para o que é importante definir sua reprodutibilidade ${ }^{(11)}$. Os coeficientes de correlação intraclasse para as cargas para 10RM, determinados por teste e reteste, foram elevados em todos os exercícios, independentemente da idade. Esses resultados podem ter sido favorecidos pelo nível de treinamento das voluntárias, pois todas tinham experiência na prática dos exercícios. A boa reprodutibilidade das cargas assegura a qualidade dos dados para a investigação dos efeitos das diferentes ordenações investigadas.

Nas mulheres jovens só não houve diferenças significativas entre SEQA e SEQB para o DP, exercício intermediário em ambas as sequências. Nas idosas a comparação intersequências revelou diferença apenas para o SH. Logo, ao menos no grupo jovem, as diferenças entre o número de repetições em SEQA e SEQB vão contra as conclusões enunciadas por Sforzo e Touey ${ }^{(25)}$. Esses autores aplicaram duas sessões de treinamento a jovens treinados do sexo masculino, uma delas iniciando-se por pequenos grupamentos musculares e a outra invertida. Os autores verificaram que, ao se iniciar o treinamento pelos grandes grupamentos musculares, a carga de trabalho tendia a ser maior, considerando-se o somatório de cargas nos exercícios.

Os resultados encontrados para as mulheres jovens aproximam-se dos que vêm sendo relatados em investigações prévias ${ }^{(12,13)}$, indicando que a musculatura solicitada no primeiro exercício de uma sequência associa-se a maior número de repetições, independentemente do tamanho do grupamento muscular envolvido. Acredita-se, nesse contexto, que se deva iniciar uma sessão de exercícios contrarresistência pelos exercícios que se deseja priorizar, não necessariamente por aqueles que acionam maiores grupamentos musculares, conforme se defende habitualmente a partir de recomendações de agências como o ACSM(26).

No caso das mulheres idosas, por outro lado, as proposições tradicionalmente veiculadas pelo ACSM com base no estudo de Sforzo e Touey ${ }^{(25)}$ foram parcialmente ratificadas. Tradicionalmente, recomendase que se inicie uma sessão de treinamento por exercícios que engagem maior massa muscular (em geral, multiarticulares), evoluindo-se para os exercícios uniarticulares ${ }^{(27)}$. A base teórica dessa recomendação decorre do entendimento de que a solicitação de maiores grupamentos musculares, no início do treinamento, estaria associada a maior volume de trabalho e demanda energética ${ }^{(28)}$. Desse modo, tais grupamentos seriam mais suscetíveis à fadiga e declínio do desempenho, uma vez precedidos por exercícios prévios. Isso se agravaria quando os exercícios uniarticulares recrutam músculos acessórios à execução dos multiarticulares, caso do TP e DP em relação ao SH no presente estudo.

Ao menos em nossa amostra de mulheres idosas, iniciar a sequência de exercícios por aquele que envolvia o maior grupamento muscular pareceu ser vantajoso, enquanto o oposto causou redução significativa no desempenho do último exercício, qual seja, o SH. Embora não se tenham aferido diretamente indicadores de fadiga, como o lactato sanguíneo, algumas razões para esses resultados podem ser especuladas. Em primeiro lugar, sabe-se que há declínio da função muscular à medida que se envelhece, que se traduz por menor potencial de geração de tensão muscular e menor tolerância à fadiga ${ }^{(3,29)}$. Desse modo, terminar a sequência pelo exercício que acarretaria maior volume de trabalho muscular, no caso das idosas, pode ter sido determinante de menor desempenho, enquanto no grupo jovem, com maior reserva, essa variável foi menos importante. Deve-se notar, além disso, que o tempo de treinamento do grupo de idosas era menor que o das mulheres jovens, o que pode ter concorrido para uma diferença ainda maior na tolerância à fadiga relacionada ao volume de trabalho associado aos exercícios propostos. Isso pode ser considerado uma limitação do estudo. Idosas e jovens tinham, respectivamente, experiência mínima com treinamento resistido de três e seis meses. Ainda assim, apenas uma idosa tinha apenas três meses de experiência, todas as demais exibindo de quatro a seis meses. De qualquer forma e apesar de três meses poderem ser suficientes para a aprendizagem dos exercícios, não é possível excluir a possibilidade de a maior experiência das jovens ter influenciado nos resultados.

Essa opinião pareceu encontrar respaldo nos resultados obtidos para a percepção subjetiva do esforço (PSE). A PSE pode ser considerada um indicador da fadiga em sessões de TCR, apesar de ser mais frequentemente utilizada como indicador de intensidade do esforço em atividades aeróbias ${ }^{(30,31)}$. De acordo com os resultados publicados por Sforzo e Touey ${ }^{(25)}$ e ratificados pelo ACSM $^{(26)}$, poder-se-ia esperar que a PSE fosse maior em sequências que se iniciam por exercícios envolvendo menores grupamentos musculares. Isso não se deu no presente estudo, principalmente nas mulheres jovens. Não foram identificadas diferenças significativas relacionadas especificamente com as ordenações propostas, em que pese o fato de envolverem apenas três exercícios. É provável que isso tenha decorrido de um volume total de trabalho similar em SEQA e SEQB, já que a escala de Borg foi aplicada somente ao final de cada sequência. O momento de aferição da PSE, ao final da terceira série das sequências, não permitiu testar a existência de diferenças especificamente relacionadas às séries e exercícios.

Por outro lado, houve incremento significativo da percepção subjetiva do esforço no grupo de idosas quando estas executaram a SEQB, ou seja, quando o exercício envolvendo o maior grupamento muscular foi realizado ao final da ordenação. Essa tendência vai ao encontro do comportamento do número de repetições, que diminuiu significativamente de TP para SH nessa sequência. Como dito, muito embora não se tenha encontrado na literatura explicações para essa diferença, não é estranho supor que esteja relacionada com a sarcopenia própria dos indivíduos que envelhecem ${ }^{(32)}$. Com menor massa muscular, pequenos grupamentos podem entrar mais facilmente em fadiga, comprometendo o rendimento dos exercícios que os utilizam como musculatura acessória. Tal é o caso, por exemplo, da passagem da TP para o SH. Isso pode ter levado a percepção subjetiva de esforço diferenciada entre as duas sequências. Em termos práticos, portanto, pode-se pensar na sugestão de Sforzo e Touey ${ }^{(25)}$, no sentido de que, para maior comodidade dos sujeitos mais velhos, dever-se-ia manter os exercícios que envolvem os grandes grupamentos musculares no início da sessão de treinamento.

É importante mencionar que são raros estudos sobre a utilização da PSE para verificar a influência da ordem de execução sobre a fadiga muscular localizada, bem como sua relação com o número de repetições ${ }^{(11-13)}$. Os resultados presentemente obtidos ratificam os de Monteiro et al. ${ }^{(11)}$ e Simão et al. ${ }^{(12)}$, que também não encontraram diferença significativa na PSE de uma sequência para outra. Por outro lado, são contraditórios aos de Simão et al. ${ }^{(13)}$, que identificaram diferenças para a PSE ao inverterem a sequência dos exercícios. 
Diferenças nos delineamentos metodológicos de cada estudo podem explicar essa contradição. Simão et al. ${ }^{(13)}$ utilizaram para prescrição do treinamento $80 \%$ de 1RM até a exaustão, sem limitar previamente o número de repetições. Nos outros estudos, inclusive o presente, o tempo de esforço tendeu a ser menor devido ao fato de os indivíduos realizarem obrigatoriamente um máximo de 10RM. O fato de em Simão et al. ${ }^{(13)}$ os indivíduos terem permanecido mais tempo executando os exercícios pode ter influenciado a sensação percebida durante o esforço. Outra diferença apresentada pelo estudo de Simão et al. ${ }^{(13)}$ refere-se ao intervalo entre as séries, que foi mantido em dois minutos, enquanto em nossa metodologia foi adotado tempo de três minutos entre as séries e exercícios. A manipulação dos intervalos pode afetar indicadores de fadiga, como o lactato sanguíneo, concentrações hormonais e reações metabólicas ${ }^{(33)}$. Assim, o intervalo menor proposto por Simão et al. ${ }^{(13)}$, juntamente com o maior trabalho mantido em cada exercício, pode ter levado a comportamento diverso da PSE. Estudos adicionais, controlando esses indicadores e variáveis potencialmente intervenientes à fadiga real e percebida, são necessários para ratificar essas opiniões.

\section{CONCLUSÃO}

Em suma, a ordem de execução de uma sequência de exercícios para membros superiores parece não afetar o volume total de trabalho realizado, mas influencia no número de repetições de cada exercício. Em mulheres jovens, o maior número de repetições tendeu a se produzir no exercício posicionado no início de uma dada se- quência. No grupo de idosas, isso se deu apenas quando a sequência se iniciou pelo menor grupamento muscular.

Em termos práticos, a ordem dos exercícios parece ser uma variável a considerar quando da elaboração de sessões para mulheres idosas, uma vez que estas responderam com maior declínio de desempenho e maior percepção do esforço que mulheres mais jovens. Isso, ao menos quando a fadiga acumulada durante a sessão é importante, caso de programas em que exercícios para segmentos próximos são feitos de maneira consecutiva (pré-exaustão, sistemas de prioridade, etc). Nas idosas, houve nítida tendência à elevação da percepção do esforço associada à sessão, quando a sequência começou pelo exercício que envolvia o menor grupamento muscular.

Pode-se pensar, portanto, que se ratifica parcialmente a tese de que o último exercício parece sofrer as maiores alterações de desempenho em virtude de fadiga acumulada durante a sessão. O estado de treinamento deveria ser levado em conta para uma previsão de desempenho nesse contexto. A percepção subjetiva de esforço pareceu refletir mais o volume total de trabalho que variações especificamente relacionadas com o desempenho em determinados exercícios e séries. Estudos adicionais são necessários para confirmar essa tendência, bem como para melhor determinar suas razões, investigando a fadiga percebida tanto ao final de sequências variadas de exercício, quanto após os próprios exercícios e séries executadas.

Todos os autores declararam não haver qualquer potencial conflito de interesses referente a este artigo.

\section{REFERÊNCIAS BIBLIOGRÁFICAS}

1. Frontera WR, Hughes VA, Krivickas LS, Kim SK, Foldvari M, Roubenoff R. Strength training in older women: early and late changes in whole muscle and single cells. Muscle Nerve 2003;28:601-8.

2. Valkeinen $\mathrm{H}$, Alen M, Hannonen P, Hakkinen A, Airaksinen O, Hakkinen K. Changes in knee extension and flexion force, EMG and functional capacity during strength training in older females with fibromyalgia and healthy controls. Rheumatology 2004;43:225-8.

3. Hunter GR, McCarthy JP, Bamman MM. Effects of resistance training on older adults. Sports Med 2004;34:329-48

4. Tak E, Staats P, Van Hespen A, Hopman-Rock M. The effects of an exercise program for older adults with osteoarthritis of the hip. J Rheumatol 2005;32:1106-13.

5. Miller MD, Crotty M, Whitehead C, Bannerman E, Daniels L. A. Nutritional supplementation and resistance training in nutritionally at risk older adults following lower limb fracture: a randomized controlled trial. Clin Rehabil 2006;20:311-23.

6. American College of Sports Medicine. Position stand. Progression models in resistance training for healthy adults. Med Sci Sports Exerc 2002;34:364-80.

7. Fleck SJ, Kraemer WJ. Fundamentos para o treinamento de força muscular. Porto Alegre: Artmed, 2006.

8. Rhea MR, Alvar BA, Burkett LN, Ball SD. A meta-analysis to determine the dose response for strength development. Med Sci Sports Exerc 2003;35:456-64

9. Wolfe BL, Lemura LM, Cole PJ. Quantitative analysis of single- vs. multiple set programs in resistance training. J Strength Cond Res 2004;18:35-47.

10. Silva NSL, Farinatti PTV. Influência de variáveis do treinamento contra-resistência sobre a força muscular de idosos: uma revisão sistemática com ênfase nas relaçōes dose-resposta. Rev Bras Med Esporte 2007;13:60-6.

11. Monteiro W, Simão R, Farinatti P. Manipulação na ordem dos exercícios e sua influência sobre número de repetições e percepção subjetiva de esforço em mulheres treinadas. Rev Bras Med Esporte 2005;11:146-50.

12. Simão R, Farinatti PTV, Polito MD, Maior AS, Fleck SJ. Influence of exercise order on the number of repetitions performed and perceived during resistive exercises. J Strength Cond Res 2005;19:152-6.

13. Simão R, Fleck SJ, Polito MD, Viveiros L, Farinatti PTV. Influence of exercise order on the number of repetitions performed and perceived exertion during resistive exercises in women. J Strength Cond Res 2007;21:23-8.

14. Macaluso A, De Vito G. Muscle strength, power and adaptations to resistance training in older people. Eur J Appl Physiol 2004;91:450-72

15. Akima H, Kano Y, Enomoto Y, Ishizu M, Okada M, Oishi Y, et al. Muscle function in 164 men and women aged 20-84 yr. Med Sci Sports Exerc 2001;33:220-6
16. Gentil P, Oliveira E, Junior VAR, Carmo J, Bottaro M. Effects of exercise order on upper-body muscle activation and exercise performance. J Strength Cond Res 2007;4:1082-6.

17. Miranda H, Fleck SJ, Simão R. Barreto AC, Dantas EHM, Novaes J. Effect of two different rest period lengths on the number of repetitions performed during resistance training. J Strength Cond Res 2007:4:1032-6.

18. Shephard RJ. PAR-Q: Canadian home fitness test and exercise screening alternatives. Sports Med 1992;5:185-95.

19. Farinatti PTV, Simão RF, Monteiro WD, Fleck, SJ. Influence of exercise order on oxygen uptake during strength training in young women. J Strength Cond Res 2008 (no prelo).

20. Baechle TR, Earle RW. Essentials of strength training and conditioning. Champaign: Human Kinetics, 2000.

21. Borg G. Perceived exertion and pain scales. Champaign: Human Kinetics, 1998

22. Sheikh K. Disability scales: assessment of reliability. Arch Phys Med Reliabil 1986;67:245-9.

23. Fleiss JL. The Design and Analysis of Clinical Experiments. New York: Wiley, 1985.

24. Bartko JJ, Carpenter WT. On the methods and theory of reliability. J Nerv Mental Dis 1976;163:307-17

25. Sforzo GA, Touey PR. Manipulating exercise order affects muscular performance during a resistance exercise training session. J Strength Cond Res 1996;10:20-4.

26. American College of Sports Medicine. Guidelines for exercise testing and prescription. Baltimore: Willians and Wilkins, 2006

27. Hass CJ, Feigenbaum MS, Franklin BA. Prescription of resistance training for healthy populations. Sports Med 2001;31:953-64

28. Hickson JF, Buono MJ, Wilmore JH, Buono MJ, Constable SH. Energy cost of weight training exercise. Natl Strength Cond Assoc 1984; $6: 22-3$.

29. Phillips WT, Haskell WL. Muscular fitness-Easing the burden of disability for elderly adults. JAPA 1995;3:261-89.

30. Day ML, Mcguigan R, Glenn B, Foster C. Monitoring exercise intensity during resistance training using the session RPE scale. J Strength Cond Res 2004;18:353-8.

31. Lagally KM, McCaw ST, Geoff GT, Medema TDQ. Ratings of perceived exertion and muscle activity during the bench press exercise in recreational and novice lifters. J Strength Cond Res 2004;18:359-64.

32. Roubenoff R, Hughes VA. Sarcopenia: current concepts. J Gerontol Med Sci 2000;55:716-24.

33. Kraemer WJ, Noble BJ, Clark MJ, Culver BW. Physiologic responses to heavy-resistance exercise with very short rest period. Int J Sports Med 1987;8:247-52. 